\title{
UNWRAPPING TECHNIQUES FOR SPECKLE PHASE MAPS: STUDY AND COMPARISON OF THE PERFORMANCE OF PRINCIPAL METHODS
}

\author{
H. M. R. Lopes ${ }^{1, a}$, J. E. Ribeiro ${ }^{1}$, M. A. P. Vaz ${ }^{2}$ and J. F. Silva Gomes ${ }^{2}$ \\ ${ }^{1}$ ESTiG - Politech. Institute of Bragança, Camp. Sta Apolónia, 5301-857 Bragança, Portugal \\ ${ }^{2}$ DEMec - Fac. of Engineering, University of Porto, R Dr. Roberto Frias, 4200-465 Porto, Portugal
}

\begin{abstract}
Holographic interferometry (HI) techniques are powerful tools for experimental mechanics which can be used for displacement fields assessment on a loaded object surface. Used with no contact and high resolution HI can be combined with image processing methods for data analysis. In these techniques the speckle should be fully resolved by the image detector to record an interferogram. This leads to noisy recordings which makes it's post-processing much difficult. Each measurement with HI ends normally in a phase map computation representing the object displacement. Phase calculation algorithms are based on spatial or temporal phase modulation and lead to wrapped phase maps. To obtain the continuous phase distribution several solutions have been implemented. This paper describes the main filtering and unwrapping algorithms available and compares it's performance to solve discontinuities in noisy recordings.
\end{abstract}

\section{Introduction}

The HI techniques are nowadays used with video recording of the interferograms. Using these techniques and image processing algorithms the object surface behaviour can be assessed by computation of the fringe pattern phase map. However, these images are exposed to several kinds of noise, like: due to speckle, from the electronic generated by numerical errors in the phase calculation. Furthermore, has to be considered the lost of image contrast as result of miss alignment of the optical setup [1]. This image represents the phase of the interferometric fringes, ranging from 0 "black" to 255 "white" and wrapped between $-\pi$ to $\pi$, for 8 bits image resolution. Due to the speckle nature, these records have always high frequency noise which can be combined with lacks of information or phase discontinuities to difficult further unwrapping process. To obtain the continuous phase distribution new filtering and unwrapping algorithms have been developed in the recent years [2-9]. They present different approaches to phase unwrapping problem, which differ in computation time, complexity and performance in dealing with phase errors and discontinuities. In experimental mechanics, the HI techniques are used for full-field measuring deformation on the surfaces of components under static or dynamic conditions. They present cost-effective advantages for nondestrutive testing (NDT) of structures [1]. In these applications high quality unwrapped phase maps are require for the evaluation of the surface behaviour. To achieve this objective, the filtering and unwrapping phase algorithms must present high noise robustness. In order to select the best

a e-mail: hlopes@ipb.pt

This is an Open Access article distributed under the terms of the Creative Commons Attribution-Noncommercial License 3.0, which permits unrestricted use, distribution, and reproduction in any noncommercial medium, provided the original work is properly cited. 
performance algorithms in these two categories, a comparative study is presented in this work based on a computed phase map.

\section{Phase maps filtering techniques}

The phase maps obtained from HI present high levels of spatial noise mainly generated by the partial or total Speckle decorrelation and by the electronics in the CCD and CMOS sensors. This high frequency noise can be reduced by using spatial filtering techniques to improve the signal/noise ratio without destroying the phase information. The nine most effective low-pass filters used in image enhancement have been select and adapted for removing the high frequency noise from phase maps. The comparative performance analysis of these filters was carried out in the same computed phase map with different noise levels added. The filters selected for this study are refereed in the literature as the most effective ones [1-12]:

- Average;

- Gaussian;

- Median;

- Wiener;

- Cubic B-Spline;

- Fast Fourier Transform (FFT);

- Windowed Fourier Transform (WFT);

- Short Time Fourier Transform (STFT);

- Wavelets transform (Wavelets);

The most common techniques for suppressing the high frequency noise use the Average and the Gaussian window filters. This filtering process is performed by applying image convolution technique, where the window size defines the smoothness level. However, this technique can't deal directly with phase discontinuities. So, the phase maps must be converted in a continues function, which can be done by transforming the phase into a complex domain $\widetilde{\phi}(x, y)=e^{i \phi(x, y)}$, where $=\sqrt{-1}$. The image convolution is then applied to the complex signals according to the expression:

$$
\tilde{\phi}^{\prime}(x, y)=\tilde{\phi}(x, y) \otimes h(m, n)
$$

where the symbol $\otimes$ represents the convolution operator and $h(m, n)$ is the selected window filter, being $m$ the horizontal and $n$ the vertical window size.

The reconstruction of the filtered phase map in spatial domain $\phi^{\prime}(x, y)$ is carried out as follow:

$$
\phi^{\prime}(x, y)=\operatorname{Tan}^{-1}\left(\tilde{\phi}^{\prime}(x, y)\right)
$$

In the median filter, the numbers doesn't follow the natural order. Thereby, the phase maps are decomposed in sine and cosine functions and then the median filters is applied to both signals:

$$
\phi^{\prime}(x, y)=\operatorname{Tan}^{-1}\left(\frac{\text { Median }\{\sin (\Delta \phi(x, y))\}}{\text { Median }\{\cos (\Delta \phi(x, y))\}}\right)
$$

The Wiener filter belongs to adaptive classes and is computed by correlating coefficients in the vicinity of each pixel $\mathrm{a}(\mathrm{x}, \mathrm{y})$. The new pixel value $b(x, y)$ is obtained from the average $\mu$ and variance coefficients $\sigma^{2}[13]$ :

$$
b(x, y)=\mu+\frac{\sigma^{2}-v^{2}}{\sigma^{2}}[a(x, y)-\mu]
$$

where $v^{2}$ is computed from the local average of variance coefficients. The Wiener filter is applied to the phase maps according to: 


$$
\phi^{\prime}(x, y)=\operatorname{Tan}^{-1}\left(\frac{\text { Wiener }\{\sin (\phi(x, y))\}}{\text { Wiener }\{\cos (\phi(x, y))\}}\right)
$$

The noise can also be removed by phase map smoothing, which can be done by adjusting Cubic BSpline to a sequence of equal space data [14]. The Cubic B-Spline coefficients are determined and the filtered phase map computed from the next expression:

$$
\phi^{\prime}(x, y)=\operatorname{Tan}^{-1}\left(\frac{\operatorname{Spline}\{\sin (\phi(x, y))\}}{\operatorname{Spline}\{\cos (\phi(x, y))\}}\right)
$$

Another approach for phase maps noise filtering are accomplish by removing the high frequency signal components, which can be performed by using Fast Fourier Transform (FFT), Windowed Fourier Transform (WFT) and Short Time Fourier Transform (STFT) techniques. Normally, the signal frequency spectrum is obtained for all the phase maps. Then, a cut-off frequency is defined for removing the high frequency signal components. Although, some signals required a more flexible approach, being the frequency spectrum computed from sub-regions of phase maps. It has the advantages of putting in evidence the high frequency signal components that can be more easily eliminated. In the WFT the filter is centred in all the image pixels while in the STFT is computed in sub-divided regions. The frequency spectrum signal of the phase map is computed:

$$
\tilde{\phi}(u, v)=\frac{1}{M N} \sum \sum \phi(x, y) e^{j 2 \pi\left(\frac{u x}{N}+\frac{v y}{M}\right)}
$$

where $u$ and $v$ are the wave number in $\mathrm{x}$ and $\mathrm{y}$ direction, respectively, for a phase map with the dimension $N \times M$. The more important signal components are preserved by applying amplitude filter $\bar{h}(u, v)$ in the spectrum domain. The filter phase map is obtained in spatial domain by using the Inverse Fast Fourier Transform:

$$
\phi^{\prime}(x, y)=\frac{1}{M N} \sum \sum \bar{h}(u, v) \times \tilde{\phi}(u, v) e^{j 2 \pi\left(\frac{u x}{N}+\frac{v y}{M}\right)}
$$

In Fast Fourier Transform the signals are converted in wave number domain and the signal spatial information is lost in this process. The WFT and STFT represent the balance between the spatial and frequency information, which the window transform the same for all the frequencies. The true representation of a signal spatial and temporal frequency requires a more flexible approach given by the Wavelets transform. The windows can vary in size and in spatial location, achieving the same accuracy representation for the signal low and high frequencies. The phase maps decomposed in 15 levels using Symlet 6 function [14]. The low amplitude components are eliminated by defining a threshold value $h$ and the filter signal is obtained using the inverse Wavelet Transform. This was performed using the Matlab ${ }^{\circledR}$ Wavelets Transform and the filter phase map is obtained according to the equation:

$$
\phi^{\prime}(x, y)=\operatorname{Tan}^{-1}\left(\frac{\text { Wavelets }^{-1}\{\text { Wavelets }[\sin (\phi(x, y))] \times h\}}{\text { Wavelets }^{-1}\{\text { Wavelets }[\cos (\phi(x, y))] \times h\}}\right)
$$

The phase maps were computed and different Gaussian noise levels have been added for the comparative performance analysis of the nine filtering techniques [15]. The generated fringes pattern with different root mean square signal to noise ratio is shown in Fig.1. It can be seen the lost of fringe contrast resulting of the increase of Gaussian noise.

The comparative performance analysis of the filtering techniques is based on minimum root mean square error between the filter phase map and theoretical pattern, determinate after optimizing technique parameters. The Fig. 2 represents the errors distribution of the nine filtering techniques for the generate phase maps. The best performance for all the maps is given by WFT and STFT techniques, with the exception of the noise-free map. 


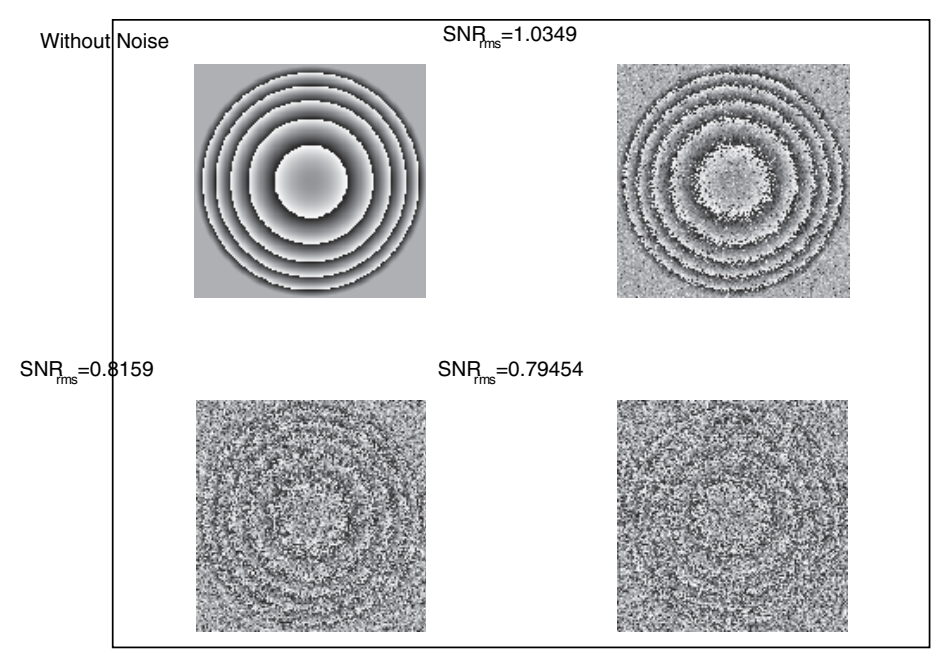

Fig. 1. The computed phase maps (256x256 pixel) with addition of different levels of Gaussian noise.

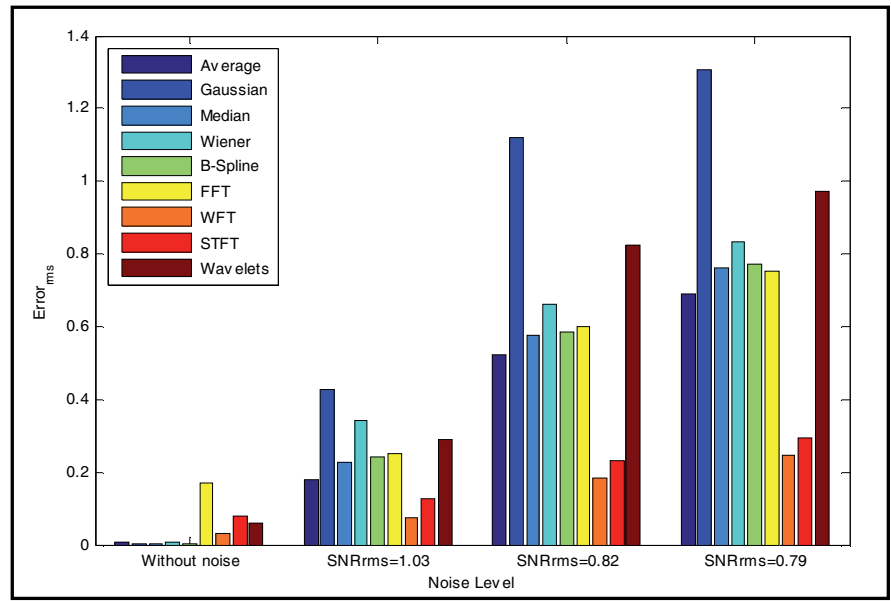

Fig. 2. The root mean square error between the filter and the original phase maps, computed for different filtering techniques.

\section{Phase unwrapping techniques}

The phase maps frequently present phase discontinues defined by the spatial amplitude modulation of the measurement field. For the amplitude reconstruction is necessary to correctly unwrapping the phase maps. The available unwrapping techniques are divided in two categories: the path-following methods and minimum-norm methods [2].

\subsection{The path-following methods}

The simplest method is based on adding or subtracting multiples of $2 \pi$ to each phase discontinuity. The integration of phase in each row is performed when the difference between consecutive pixel is higher than $-\pi$ or $\pi$, being added or subtracted $2 \pi$ to the remain row pixels. The process ends by repeating the same procedure for each column. However, the presence of phase inconsistencies and phase ambiguities lead to non-unique solutions. The phase inconsistencies are represented by interruptions in fringe pattern and/or discontinuities on the phase map, which are 
called by poles or phase residues.

To assure that the phase map integration is unique, new methods have proposed the introduction of restriction lines to avoid this phase inconsistencies. Four methods have distinguished using this approach [2]. They are ordered by complexity degree:

- Goldstein;

- Quality-Guided;

- Mask Cut; and

- Flynn;

The Goldstein method [16] is base on the potential equilibrium of the poles and in the introduction of restriction lines to the integration path of the phase map.

The Quality-Guided method uses the information about the distribution of the phase quality to define the phase integration path. The phase quality map is determined starting from a threshold value of the phase map second order derivative [17, 18].

The combination of this two concepts, phase quality distribution and restriction lines, conduced to a hybrid method, designated by Mask Cut method [19, 20]. It combines the advantages of the two previous methods, having the quality phase to put the restriction lines and minimizing the mistakes during integration of the phase.

Finally, the Flynn method [21] is based on the definition on transition frontiers that represent phase transition of $-\pi$ or $\pi$, dividing the map in regions. The phase integration process is accomplished by minimizing the phase transitions between regions.

\subsection{The minimum-norm methods}

The approach of these methods is based on the minimization problem, designated by error minimum-norm, and defined as the difference between the spatial derivatives of unwrapped phase and the derivatives of the phase map [2].

The objective function can be formulated in terms of weighty or non-weighty distribution of the error norm [2]. This leads to the definition of three methods for the resolution of the minimization problem:

- FFT/DCT;

- PCG;

- Multigrid;

For the non-weighty formulation of the error norm, the solution of the minimization problem is reduced to the resolution of a system of discreet Poisson equations [22]. The Fast Fourier Transform (FFT) or Discreet Cosines Transform (DCT), allows the simple and efficient resolution of the linear equations deduced from Poisson equations. The method here adopted uses direct and inverse Fourier transform with extension of the information beyond the image, created by mirroring the image [23].

The weighty formulation introduce, in relation to the previous method, the derived weights of the methods of Quality-Guided and Mask Cut to improve the integration of the phase and isolate the regions with low signal to noise ratio. The Preconditioned Conjugate Gradient method is used to efficiently determine the solution of the minimization problem [2].

The Multigrid iteration methods are used for the fast resolution of large scale partial or non-linear systems. These methods incorporate the Gauss-Seidel relaxation process to increase the convergence tax. The computational time of this method for large scale problems can be up to 25 times faster than the PCG method [2].

The evaluation of the unwrapping phase methods performance is based in the analysis of two phase maps shown in Fig. 3. Several phase inconsistencies and discontinuities were introduced in this map, under the form of interruptions in the phase transition contour, reflecting the two of the most common causes. 


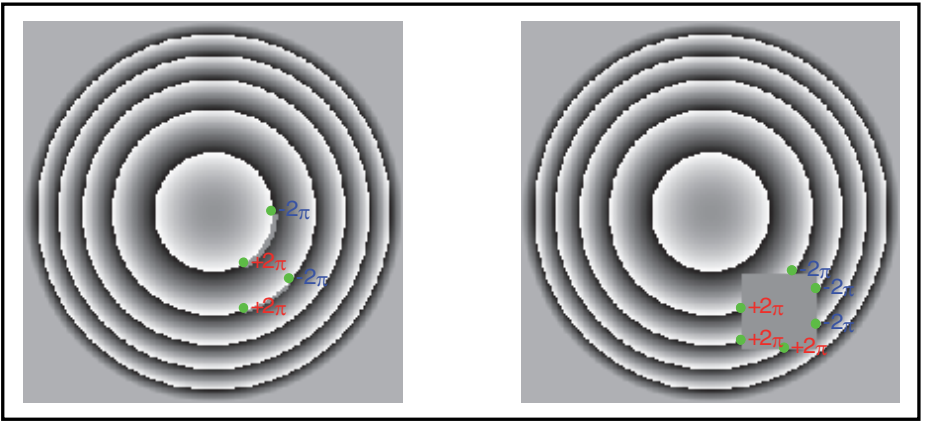

Fig. 3. Phase maps with: phase transition inconsistencies (left); phase map discontinuities (right),

In order to determine the most effective methods the comparative analysis of the differences is accomplished among the continuous maps obtained by application of these methods and the simulated continuous map. The Fig. 4 and 5 represents the unwrapping phase deviation for the 8 methods in analysis.

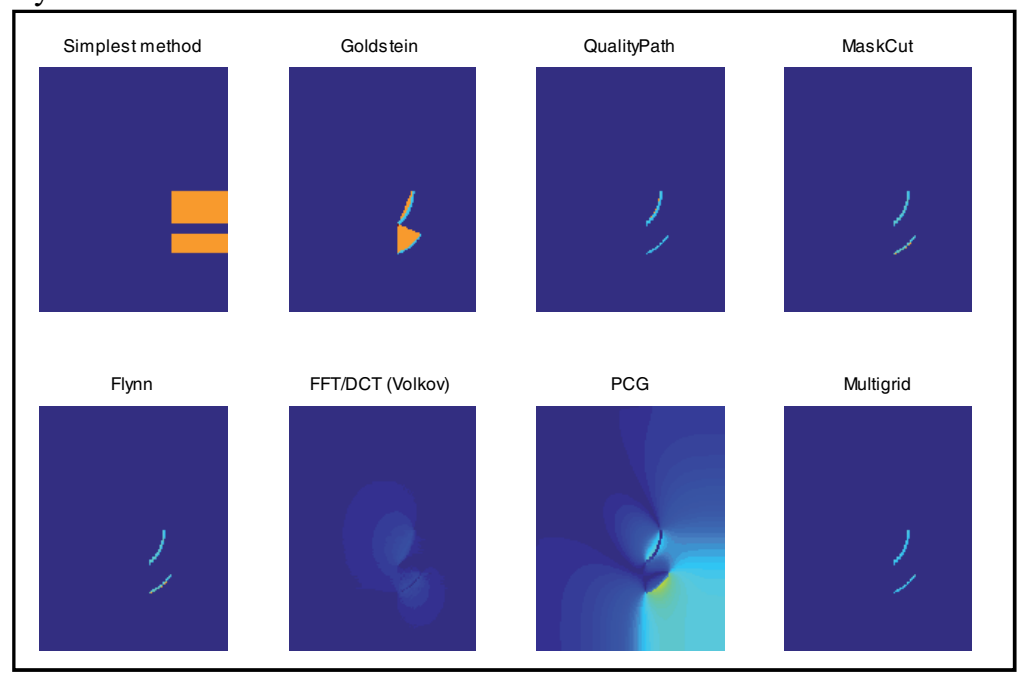

Fig. 3. Deviations in unwrapping phase methods due to a phase inconsistencies in the phase map (Fig. 3 left).

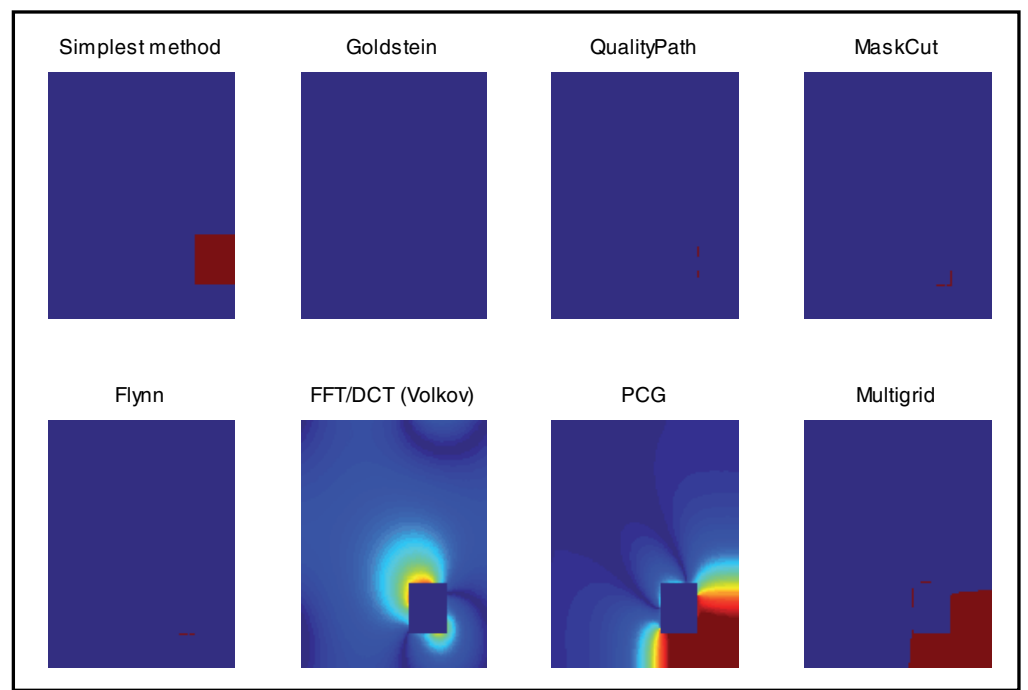

Fig. 4. Deviations in unwrapping phase methods due to discontinuity in the phase map (Fig. 3 right). 
The comparative analysis of these results reveals that, in general, the path-following methods present the deviation limited to the phase inconsistencies, close to the areas where restriction lines are placed, while the minimum-norm methods, they spread these inconsistencies to the neighbouring areas. This effect is more evident when phase inconsistencies are spread in large areas. These results allows saying that the minimum-norm methods are more effective in the presence of large quantities of phase inconsistencies and the path-following methods are better behaved for the local discontinuities.

\section{Experimental applications}

A commercial HI system use a LUMONICS Ruby laser generates pairs of pulses with a time separation between 1 and $800 \mathrm{~ms}$ [24]. A spatial carrier is introduced in the primary fringes to perform the phase calculation [1]. This technique was chosen because it produces highly noisy phase maps.

A beam was suspended by elastic bands and excited acoustically to its natural frequencies by a loudspeaker mounted close to its backside surface. The two first mode shapes were measured using this system and the corresponding interferograms were pos-processed to obtain the phase maps shown Fig. 5.

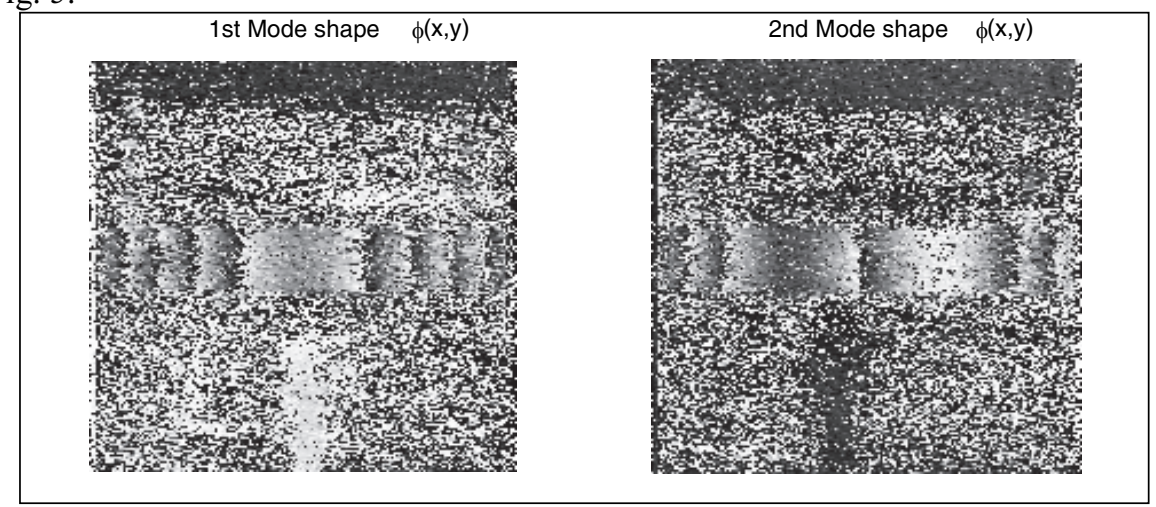

Fig. 5. The phase maps of the two beam mode shapes.

The unwrapped phase maps of the two natural frequencies represented in Fig.6 were obtained using filtering technique WFT and the unwrapping method Flynn.

The comparative analysis between the experimental measurements and the numeric simulation reveals that the results are very close. This proves the effectiveness of the application of the selected methods to filtering and unwrapping the experimental phase maps.

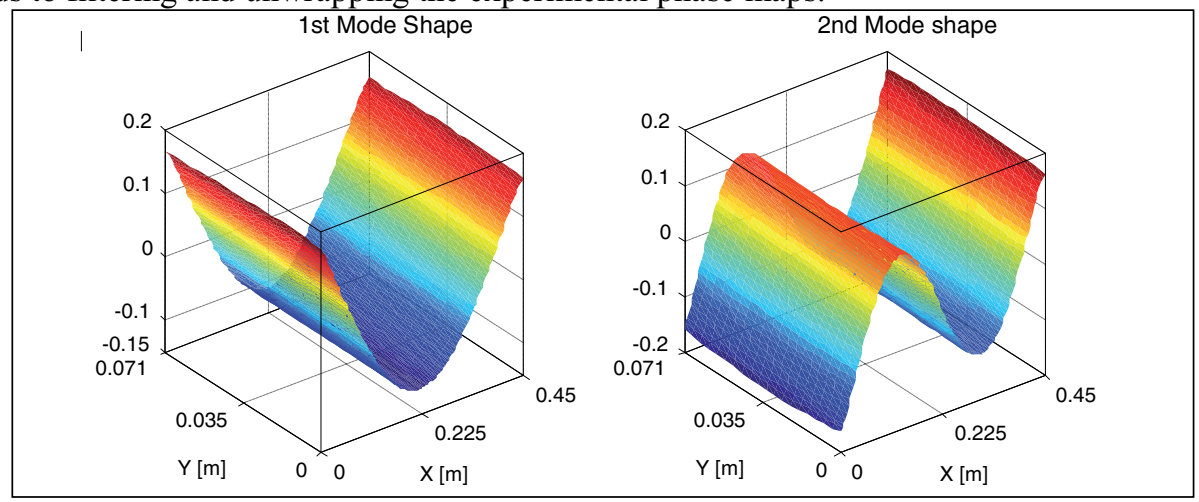

Fig. 6. The first two beam mode shapes. 


\section{Conclusions}

The filtering techniques showed to be very effective in the reduction of the experimental noise and they constitute an efficient way to improve the quality of the experimental results. The WFT and STFT techniques present a superior performance in filtering the phase maps noise from. The unwrapping methods allow removing the phase discontinuities. However, this process becomes complex in the presence of the phase inconsistencies. For the resolution of this problem two different strategies are proposed: path-following methods and minimum-norm methods. The first ones are shown to be particularly effective in the resolution of concentrated phase inconsistencies in a small area, while the second methods possess a better performance in the resolution of random spread inconsistencies. However, the classification of the phase maps in these two categories is not always easy. In these cases, the most suitable procedure is to evaluate the individual acting of these techniques. Finally, the methods with best performance can efficiently solve the noisy experimental phase maps.

\section{References the level of}

1. Kreis, T., Handbook of holographic interferometry : optical and digital methods. Weinheim: Wiley-VCH. xii, $542 \mathrm{p}$ (2005)

2. Ghiglia, D.C. and M.D. Pritt, Two-dimensional phase unwrapping : theory, algorithms, and software. New York: Wiley. xiv, 493 p (1998)

3. Aebischer, H.A. and S. Waldner, A simple and effective method for filtering speckle-interferometric phase fringe patterns. Optics Communications, 162(4-6): p. 205-210 (1999)

4. Asundi, A. and W. Jun, Strain contouring using Gabor filters: principle and algorithm. Optical Engineering, 41(6): p. 1400-1405. (2002)

5. Jun, W. and A. Asundi, Strain contouring with Gabor filters: filter bank design. Applied Optics, 2002. 41(34): p. 7229-7236. (2002)

6. Kemao, Q., Seah Hock Soon, Anand Assundi, A Simple phase unwrapping approach based on filtering by windowed Fourier transform. Optics \& Laser Technology, 37: p. 458-462. (2005)

7. Kemao, Q., Windowed Fourier transform for fringe pattern analysis. Applied Optics, 2004. 43(13): p. 2695-2702.

8. Volkov, V.V. and Y.M. Zhu, Deterministic phase unwrapping in the presence of noise. Optics Letters, 28(22): p. 2156-2158. (2003)

9. Kemao, Q., Windowed Fourier transform for fringe pattern analysis: addendum. Applied Optics, 2004. 43(17): p. 3472-3473.

10. Reinsch, C., Citation Classic - Smoothing by Spline Functions. Current Contents/Engineering Technology \& Applied Sciences, 24: p. 20-20. (1982)

11. Aebischer, H.A. and S. Waldner, A simple and effective method for filtering speckle-interferometric phase fringe patterns. Optics Communications 162(4-6): p. 205-210 1999

12. Jun, W. and A. Asundi, Strain contouring with Gabor filters: filter bank design. Applied Optics, 41(34): p. 7229-7236 (2002).

13. Lim, J.S., Two-Dimensional Signal and Image Processing. Prentice Hall. (1990)

14. Daubechies, I., Ten lectures on wavelets, ed. SIAM. (1992)

15. Servin, M. and F.J. Cuevas, A noval technique for spatial phase-phifting interferometry. J. Mod. Opt., 42: p. 1853-1862. (1995)

16. Goldstein, R.M., R.H.A. Zebker, and C.L. Werner, Satellite radar interferometry: two dimensional phase unwrapping. Radio Science, 23(4): p. 713-720. (1988)

17. Bone, D.J., Fourier Fringe analysis: the two-dimensional phase unwrapping problem. Appl Opt, 30(25): p. 3627-3632. (1991)

18. Xu, Y. and C. Ai. Simple and effective phase unwrapping technique. in Interferometry IV: Techniques and Analysis. p. 254-263, Bellingham: SPIE. (1993)

19. Prati, C., M. Giani, and N. Leuratti. SAR interferometry: A 2-D phase unwrapping technique based on phase and absolute values information. in International Geoscience and Remote Sensing Symposium. p. 2043-2046, IEEE. (1990)

20. Flynn, T.J. Consistent 2-D phase unwrapping guided by a quality map. in International Geoscience and Remote Sensing Symposium. p. 2057-2059, IEEE. (1996) 
21. Flynn, T.J., Two-dimentional phase unwrapping with minimum weighted discontinuity. Journal of the Optical Society of America A, 14(10): p. 2692-2701. (1997)

22. Song, S.M., S. Napel, N.J. Pelc, and G.H. Glover, Phase Unwrapping of MR Images Using Poisson Equation. IEEE Trans Imag Process., 4: p. 667-676. (1995)

23. Volkov, V.V. and Y.M. Zhu, Deterministic phase unwrapping in the presence of noise. Optics Letters, 28(22): p. 2156-2158. (2003)

24. Ettemeyer.GmbH\&Co., ESPI Pulse. (1999) 